\title{
Modeling the diffuse X-ray emission of planetary nebulae with different chemical composition
}

\author{
Matthias Steffen, Christer Sandin, Ralf Jacob, \\ and Detlef Schönberner
}

Leibniz-Institut für Astrophysik Potsdam (AIP),

An der Sternwarte 16, 14482 Potsdam, Germany

\begin{abstract}
Based on time-dependent radiation-hydrodynamics simulations of the evolution of Planetary Nebulae (PNe), we have carried out a systematic parameter study to address the non-trivial question of how the diffuse X-ray emission of PNe with closed central cavities is expected to depend on the evolutionary state of the nebula, the mass of the central star, and the metallicity of stellar wind and circumstellar matter. We have also investigated how the model predictions depend on the treatment of thermal conduction at the interface between the central 'hot bubble' and the 'cool' inner nebula, and compare the results with recent X-ray observations. Our study includes models whose properties resemble the extreme case of PNe with Wolf-Rayet type central stars. Indeed, such models are found to produce the highest X-ray luminosities.
\end{abstract}

Keywords. planetary nebulae: general, X-rays: stars, stars: winds, hydrodynamics, conduction

\section{Introduction}

Over the past decade, roughly a dozen nearby Galactic Planetary Nebulae were found to be a source of extended diffuse X-ray emission (e.g. Kastner et al. 2008; Kastner 2009). Images taken from space by the X-ray observatories Chandra and XMM-Newton reveal that the diffuse $\mathrm{X}$-ray emission is confined to the closed central cavities of round and elliptical PNe, indicating the presence of hot gas with temperatures in excess of $10^{6} \mathrm{~K}$ (for recent examples see Guerrero et al., this volume). Basically, these findings confirm theoretical predictions of wind-heated 'hot bubbles', even though the observed X-ray temperatures are much lower (at least a factor of 10) than obtained from simple spherical models assuming that the wind kinetic energy is thermalized in an adiabatic shock. However, more elaborate models taking into account the heat flux due to thermal conduction across the central 'hot bubble', show much lower X-ray temperatures, in good agreement with observational evidence (Steffen et al. 2008). About $75 \%$ of the targeted PNe show no evidence of diffuse X-rays, among them old, evolved nebulae and objects with open structures. Note that a large fraction of PNe also harbor X-ray point sources at the position of their central stars. This type of X-ray emission, however, is of a different nature than the extended X-ray emission from the shock-heated hot wind bubbles which are the subject of the present study.

The number of PNe with detected diffuse X-ray emission is too small to allow the derivation of reliable empirical trends, such as relations between stellar wind parameters and X-ray properties of the nebula. Likewise, it is difficult to make theoretical predictions based on simple back-of-the-envelope calculations. In very general terms, the X-ray luminosity, $L_{\mathrm{X}}$, is given by the X-ray emissivity per unit mass and electron density, $\eta$, integrated over the emitting volume: $L_{\mathrm{X}}=\bar{\eta}\left(T_{\mathrm{e}}, Z\right) M_{\mathrm{X}} \overline{n_{\mathrm{e}}} \sim \bar{\eta}\left(T_{\mathrm{e}}, Z\right) M_{\mathrm{X}}^{2} R_{\mathrm{X}}^{-3}$. The emissivity depends on the electron temperature, $T_{\mathrm{e}}$, and the metallicity of the plasma, $Z$; the 
mean electron density is proportional to the X-ray emitting mass, $M_{\mathrm{X}}$, divided by the X-ray emitting volume, $\overline{n_{\mathrm{e}}} \sim M_{\mathrm{X}} R_{\mathrm{X}}^{-3}$. If we want to know how the X-ray luminosity of an object changes in the course of its evolution, we have to know how the product $M_{\mathrm{X}}^{2} R_{\mathrm{X}}^{-3}$ changes with time. This depends mainly on the time evolution of the fast wind of the central star: for a constant wind expanding into an environment with a radial density profile $\rho \propto r^{-2}$, it can be shown that $M_{\mathrm{X}} \propto t$, and $R_{\mathrm{X}} \propto t$ (Koo \& McKee 1992), such that the X-ray luminosity decreases as $L_{\mathrm{X}} \propto t^{-1}$. On the other hand, the more realistic assumption that wind power and momentum flux increase with time, e.g. $\dot{M} V^{2} \propto t^{2.62}$, $\dot{M} V \propto t^{1.02}$, leads to an increasing X-ray luminosity, $L_{\mathrm{X}} \propto t^{1.2}$ (Zhekov \& Perinotto 1996). Moreover, properties of the X-ray emission also depend on the thermal structure of the hot plasma, which is ruled by the efficiency of electron heat conduction. Clearly, detailed numerical models are necessary to make reliable theoretical predictions.

\section{Model calculations}

Using the Potsdam NEBEL code, we model the combined evolution of central star (CS) and circumstellar envelope by radiation hydrodynamical simulation in 1D spherical geometry, accounting for non-equilibrium ionization of the nine most important chemical elements. A typical model has a radial extent from $6 \times 10^{14}$ to $3 \times 10^{18} \mathrm{~cm}(0.0002$ to $1.0 \mathrm{pc}$ ). Treated in a physically consistent way, it contains the freely expanding CS wind, the inner reverse shock, the 'hot bubble' consisting of the shocked stellar wind, and the (double-shell) PN proper, separated from the 'hot bubble' by the contact discontinuity and from the surrounding AGB halo by the outer shock. Temporally, the simulations start at the tip of the AGB and are advanced well into the white dwarf regime, thus covering the formation and complete evolution of the PN.

Given the radial temperature and density structure of the hydrodynamical model, we apply the Chianti code to compute, for selected instants along the evolutionary sequence, the emergent X-ray spectrum, the X-ray luminosity, $L_{\mathrm{X}}$, the characteristic X-ray temperature, $T_{\mathrm{X}}$, and the surface brightness distribution of the X-ray emitting hot central cavity. For details about the model calculations see Steffen et al. (2008) and references therein.

\section{Simulated X-ray emission: a parameter study}

The work by Steffen et al. (2008) was restricted to models with standard Galactic-disk elemental abundances, as given in their Table 1 . The present parameter study considers the influence of the adopted chemical composition on the resulting X-ray properties.

Models with scaled metallicities. We have obtained model sequences of different metallicity, $Z$, by globally scaling the number densities (relative to $\mathrm{H}$ ) of the 'metals' $\mathrm{C}, \mathrm{N}$, $\mathrm{O}, \mathrm{Ne}, \mathrm{S}, \mathrm{Cl}$, and $\mathrm{Ar}$ by a common factor $f_{Z}$ with respect to the standard Galacticdisk abundance mix, $Z=f_{Z} Z_{\mathrm{GD}}$. As explained in detail in Schönberner et al. (2010), changes of the metal content affect both the cooling properties of the nebular matter (higher $Z \Rightarrow$ more efficient line cooling), and the wind of the central star (higher $Z \Rightarrow$ higher wind power). Specifically, we assume here $\dot{M} \propto Z^{0.69}$ and $V_{\text {wind }} \propto Z^{0.13}$, so that $L_{\text {wind }} \equiv 1 / 2 \dot{M} V_{\text {wind }}^{2} \propto Z^{0.95}$. At the same time, we assume that the evolution of the central star, $T_{\text {eff }}(t), L_{*}(t)$, is unaffected by changes of $Z$ (see Schönberner et al. 2010).

Our previous studies at standard Galactic-disk composition, based on models including heat conduction, had shown that the X-ray luminosity increases steadily with time $\left(T_{\text {eff }}\right)$ until the turnaround point (maximum $T_{\text {eff }}$ ) is reached, after which $L_{*}, L_{\text {wind }}$, and $L_{\mathrm{X}}$ drop rapidly. Essentially, the time evolution of $L_{X}$ reflects the evolution of $L_{\text {wind }}$. The so far unexplored metallicity dependence of $L_{\mathrm{X}}$ and $T_{\mathrm{X}}$ is shown in Fig. 1 for a central star with $M_{*}=0.595 \mathrm{M}_{\odot}$. For given $T_{\text {eff }}, L_{\mathrm{X}}$ increases systematically with metallicity (left 

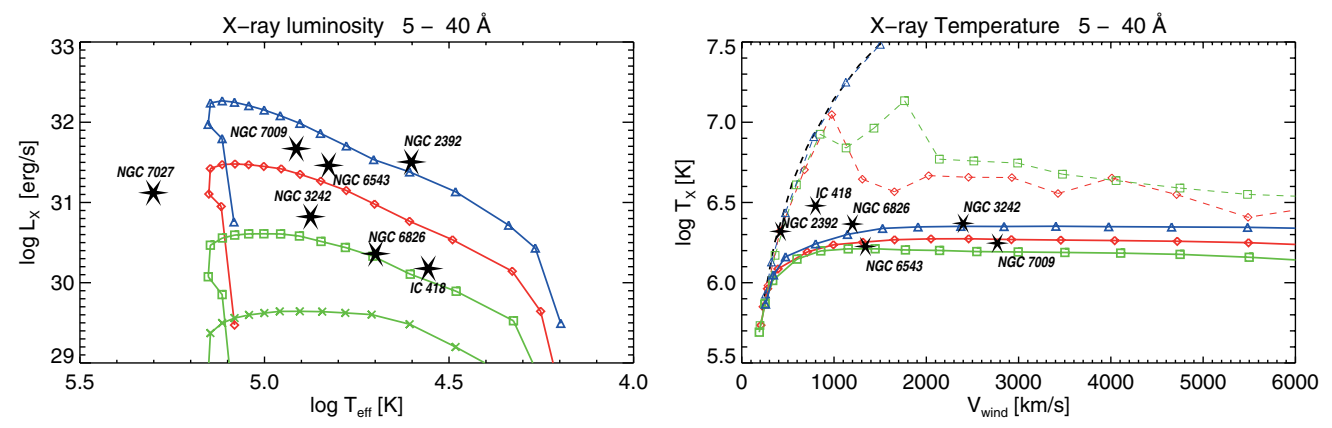

Figure 1. Evolution of the X-ray luminosity, $L_{\mathrm{X}}$, as a function of the CS effective temperature (left), and of the X-ray temperature, $T_{\mathrm{X}}$, as a function of the CS wind speed (right), for a central star with $M_{*}=0.595 \mathrm{M}_{\odot}$. The solid curves connecting data points (symbols) refer to model sequences computed with heat conduction HC2 (see), assuming metallicity scaling factors of $f_{Z}=3$ (triangles), 1 (diamonds), $1 / 3$ (squares), and $1 / 10$ (crosses). Corresponding sequences computed without heat conduction are shown by the dashed curves (right panel only), omitting the data points for $f_{Z}=3, V_{\text {wind }}>2000 \mathrm{~km} / \mathrm{s}$ which are numerically uncertain. Stars mark the position of observed PNe with normal (H-rich) chemical composition (data M. Guerrero 2011, priv. comm.). $L_{\mathrm{X}}$ and $T_{\mathrm{X}}$ refer to the X-ray emission between 5 and $40 \AA$ ( 0.3 to $\left.2.5 \mathrm{keV}\right)$.

panel). Naively, one might have expected that roughly $L_{\mathrm{X}} \propto Z^{2}$, since both $L_{\text {wind }}$ and the number of metal ions scale with $Z$. The numerical results, however, show a weaker $Z$ sensitivity, $L_{\mathrm{X}} \propto Z^{p}, 1<p<2$, where $p$ depends on $T_{\text {eff }}$. The X-ray temperature increases only slightly with metallicity (right panel), in quantitative agreement with the scaling suggested by Eq. (14) in Steffen et al. (2008), noting that $L_{\text {wind }} \propto Z$. In the absence of heat conduction, $T_{\mathrm{X}}$ is significantly higher (Fig. 1, right panel) and - except for the earliest stages of evolution $-L_{\mathrm{X}}$ is reduced by roughly 2 orders of magnitude (not shown).

We have also investigated sequences of different metallicity for $M_{*}=0.625 \mathrm{M}_{\odot}$ and $0.696 \mathrm{M}_{\odot}$. Qualitatively, the $Z$ dependence of the $\mathrm{X}$-ray properties is very similar to the case $M_{*}=0.595 \mathrm{M}_{\odot}$. Summarizing the combined $Z, M_{*}, t$ dependence, the models predict the highest $L_{\mathrm{X}}$ for metal-rich nebulae with evolved (hot) massive central stars.

Models with Wolf-Rayet-type central stars. Wolf-Rayet (WR) type central stars are characterized by a hydrogen-deficient chemical composition of their atmospheres and winds, and by mass loss rates that exceed those of normal central stars by factors 10 to 100. In a first attempt to model the X-ray emission of PNe with WR-type central stars, we have modified our standard simulation $\left(M_{*}=0.595 \mathrm{M}_{\odot}\right)$ in two respects. First, we changed the chemical composition of the CS wind from standard (H-rich) to WR-type $(2 \% \mathrm{H}, 42 \% \mathrm{He}, 49 \% \mathrm{C}, 5 \% \mathrm{O}$ by mass) at the tip of the AGB $(t=0)$. This implies that the 'hot bubble' forms out of H-deficient matter, while the surrounding nebula remains $\mathrm{H}$ rich. For consistency, we have updated the NEBEL code with a generalized description of electron heat conduction that is valid for arbitrary chemical composition (Sandin et al., this volume). The impact of changing the chemistry of the CS wind is shown by the difference between the two lower curves in Fig. 2 (left panel). The H-deficient 'hot bubble' forms at $t=1340 \mathrm{y}, T_{\text {eff }}=15.4 \mathrm{kK}, V_{\text {wind }}=260 \mathrm{~km} / \mathrm{s}$, slightly later than the H-rich bubble $(1190 \mathrm{y}, 13.1 \mathrm{kK}, 165 \mathrm{~km} / \mathrm{s})$, but reaches much higher X-ray luminosities (up to a factor 100) during the early evolution (low $\left.T_{\text {eff }}\right)$. Obviously, the X-ray emissivity (per unit mass) is much higher for WR-type matter. Later, the difference in $L_{\mathrm{X}}$ tends to vanish, since the bulk of the hot gas is increasingly made up of evaporated H-rich matter (see below). As a second step, we have increased the CS mass loss rate globally by a factor 100 . This delays the formation of the 'hot bubble' both for normal $(1620 \mathrm{y}, 19.3 \mathrm{kK}, 325 \mathrm{~km} / \mathrm{s})$, and WR-composition CS winds $(2160$ y, $25.4 \mathrm{kK}, 720 \mathrm{~km} / \mathrm{s})$. As seen in Fig. 2 (left, upper two 

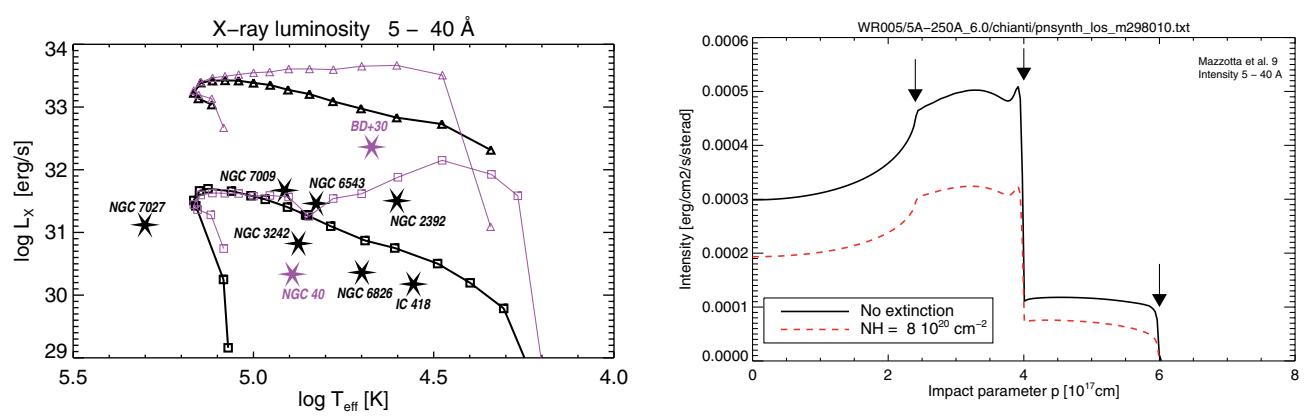

Figure 2. Left: Evolution of $L_{\mathrm{X}}$ over CS effective temperature, for $M_{*}=0.595 \mathrm{M}_{\odot}$. The two thick curves refer to model sequences with standard chemical composition, the corresponding thin curves show the effect of changing the CS wind chemistry from standard (H-rich) to WRtype (H-deficient) at approximately identical $\dot{M}(t), V_{\text {wind }}(t)$, and unchanged H-rich composition of the nebula. The upper two tracks (triangles) have $\dot{M}(t)$ increased by a factor $100\left(V_{\text {wind }}(t)\right.$ unchanged) with respect to the standard mass loss rate used with the lower two tracks (squares). All sequences have been computed with heat conduction HC2 (see Steffen et al. (2008)). The observed positions of two PNe with WR-type central stars are also indicated (stars marked BD $+30^{\circ} 3639$ and NGC 40; data M. Guerrero 2011, priv. comm.). Right: Synthetic X-ray surface brightness profile computed for the uppermost track in the left panel at $T_{\text {eff }} \approx 90000 \mathrm{~K}$. From left to right, arrows indicate the position of the inner shock, the chemical discontinuity, and the conduction front. Both plots refer to the X-ray emission between 5 and $40 \AA$ ( 0.3 to $2.5 \mathrm{keV})$.

curves), such models easily exceed the X-ray luminosity measured for $\mathrm{BD}+30^{\circ} 3639$, a well studied PN with a [WC]-type central star. Again, corresponding models calculated without heat conduction typically have lower $L_{\mathrm{X}}$ by roughly a factor 100, except at the very early stages where heat conduction is negligible. Finally, Fig. 2 (right) shows the X-ray surface brightness distribution of the test model with WR-type wind composition and scaled-up mass loss rate at a late stage of evolution $\left(T_{\text {eff }} \approx 90000 \mathrm{~K}\right)$. The 'hot bubble' is divided into two chemically distinct parts: the inner region $\left(r \leqslant 4 \times 10^{17} \mathrm{~cm}\right)$ consists of $\mathrm{H}$-deficient matter, the outer shell consists of $\mathrm{H}$-rich matter that evaporated due to heat conduction. In X-rays, the H-deficient core of the central cavity shines much brighter than the surrounding H-rich layer (cf. Jacob et al., this volume).

\section{Conclusions}

The observed X-ray luminosities of PNe with normal H-rich composition can be explained by hydrodynamical models with heat conduction, either assuming a standard Galactic-disk metallicity, $Z_{\mathrm{GD}}$, and a range of central star masses between $M_{*}=0.565$ and $0.7 \mathrm{M}_{\odot}$, or by a fixed $M_{*}=0.625 \mathrm{M}_{\odot}$ and a range of metallicity scaling factors between $f_{Z}=1 / 3$ and $f_{Z}=3$. The highest X-ray fluxes are expected to originate from $\mathrm{PNe}$ with WR-type central stars, due to their powerful and H-deficient winds. Models with suppressed thermal conduction (magnetic fields) may explain X-ray non-detections.

\section{References}

Kastner, J. H., Montez, R., Balick, B., \& De Marco, O. 2008, ApJ, 672, 957

Kastner, J. 2009, in: S. Wolk, A. Fruscione, D. Swartz (eds.), Chandra's First Decade of Discovery, http://cxc.harvard.edu/symposium_2009/proceedings/session_01.html\#talk03

Koo, B.-C. \& McKee, C. F. 1992, ApJ, 338, 103

Schönberner, D., Jacob, R., Sandin, C., \& Steffen, M. 2010, A\&A, 523, A86

Steffen, M., Schönberner, D., \& Warmuth, A. 2008, $A \mathscr{E} A$, 489, 173

Zhekov, S. A. \& Perinotto, M. 1996, A\& A, 309, 648 